\title{
Per-oral endoscopic dual myotomy for the treatment of achalasia
}

\author{
Xianglei Yuan ${ }^{1} \cdot$ Zhe Feng $^{1} \cdot$ Yanshi Zhao $^{2} \cdot$ Xianhui Zeng $^{1} \cdot$ Liansong Ye $^{1} \cdot$ Wei Liu $^{1} \cdot$ Bing Hu$^{1}$
}

Received: 20 April 2021 / Accepted: 12 July 2021 / Published online: 17 July 2021

(c) The Author(s) 2021

\begin{abstract}
Background Repeat per-oral endoscopic myotomy is occasionally performed for persistent/recurrent symptoms in patients with achalasia, and yields favorable outcomes. We investigated a novel technique, per-oral endoscopic dual myotomy (dualPOEM), where a second myotomy was performed during a single session to augment the efficacy and avoid repeat interventions. The aim of this study was to evaluate its feasibility, safety and efficacy.

Methods Consecutive patients diagnosed with achalasia who underwent dual-POEM (1/2018-5/2019) were prospectively collected and retrospectively analyzed. Patients with baseline Eckardt score $\geq 9, \geq 10$ years of symptoms, and/or having prior interventions other than myotomy received dual-POEM. The primary outcome was clinical success (Eckardt score $\leq 3$ ). Secondary outcomes were procedure-related adverse events, change in lower esophageal sphincter (LES) pressure, and reflux complications.

Results Seventeen patients received dual-POEM. Procedure-related adverse events were observed in 2 (11.8\%) patients (mucosal injury and pneumonitis). Both were minor in severity. During a median follow-up of 33 months (interquartile range, IQR [31,35]; range, 19-36), clinical success was achieved in 16 (94.1\%) patients. The median Eckardt score decreased from 9 (IQR [8, 11.5]; range 7-12) to 1 (IQR [1, 2]; range 0-4) $(P<0.001)$, and LES pressure decreased from $25.8 \mathrm{mmHg}$ (IQR [21.7,33.5]; range 17.7-46.3) to 7.4 $\mathrm{mmHg}(\mathrm{IQR}[6.3,10.4]$; range 2.2-12.6) $(P<0.001)$. Seven $(41.2 \%)$ patients developed postprocedural reflux either by gastroesophageal reflux disease questionnaire or esophagitis endoscopically, all successfully treated with proton pump inhibitors.

Conclusion Dual-POEM preliminarily demonstrated high efficacy with a favorable safety profile in patients with achalasia with predictors of treatment failure.
\end{abstract}

Keywords Achalasia $\cdot$ Endoscopic treatment $\cdot$ Myotomy $\cdot$ Dual

\section{Introduction}

Achalasia is a primary neurodegenerative disorder of the esophagus characterized by incomplete lower esophageal sphincter (LES) relaxation and absence of esophageal body peristalsis [1]. The pathogenesis of achalasia is incompletely understood; thus, treatment mainly focuses on relaxation

Xianglei Yuan and Zhe Feng are co-first authors.

Bing $\mathrm{Hu}$

hubingnj@163.com

1 Department of Gastroenterology, West China Hospital, Sichuan University, No. 37 Guo Xue Alley, Chengdu 610041, Sichuan, China

2 Division of Gastroenterology, Hepatology and Nutrition, Department of Medicine, Loma Linda University, Loma Linda, USA or mechanical disruption of the LES to relieve symptoms. Conventional treatment modalities include pharmacologic agents, botulinum toxin injection, balloon dilation and laparoscopic Heller myotomy (LHM) [1]. Inspired by the concepts of natural orifice transluminal endoscopic surgery, peroral endoscopic myotomy (POEM), creating a submucosal tunnel as the operative space for myotomy, was described and first performed in human patients in 2010 [2]. Subsequently, due to its safety and high efficacy [3, 4], POEM has been used as the first-line treatment for achalasia in many centers across the world.

Despite the remarkable success of POEM, a percentage of patients have persistent or recurrent symptoms afterwards and require additional interventions [5-9]. Although the exact reasons for POEM failure remain largely unknown, an incomplete myotomy (namely insufficient gastric myotomy) is considered to be one of the most likely causes [ 8 , 
9]. In addition, some risk factors for POEM failure, including higher pretreatment Eckardt score $(\geq 9)$, long disease duration ( $\geq 10$ years), history of previous interventions, and submucosal fibrosis have also been reported $[5,6,8$, 9]. Multiple studies indicated that repeat myotomy can be performed with favorable outcomes in patients with such risk factors after their initial POEM failure [5, 7, 9, 10]; but it increased the health care costs and delayed the time to remission. To avoid repeat interventions in patients with risk factors for POEM failure, we investigated the performance of a technique, per-oral endoscopic dual myotomy (dualPOEM) [11], where dual myotomy was performed during a single procedure. Here, we conducted a retrospective study and aimed to evaluate the feasibility, safety and efficacy of dual-POEM for patients with achalasia who have risk factors of POEM failure.

\section{Methods}

\section{Patients}

In this retrospective study, consecutive patients with achalasia who underwent dual-POEM from January 2018 to May 2019 at West China Hospital of Sichuan University, were included. We reviewed the clinical data of included patients from our prospectively collected database. Patients without available follow-up data were excluded. This study protocol was approved by the Ethics Committee on Biomedical Research, West China Hospital of Sichuan University. Informed consent was obtained from all involved patients.

\section{Dual-POEM procedure and follow-up protocol}

Patients who met at least one of the following criteria underwent dual-POEM: (1) preoperative Eckardt score $\geq 9$; (2) long disease duration $\geq 10$ years; (3) and prior interventions other than myotomy. All procedures were performed by a single experienced POEM endoscopist ( $>300$ POEM procedures). Patients were given a liquid diet for 2 days and fasted for at least $8 \mathrm{~h}$ before dual-POEM. Antibiotics were given intravenously $30 \mathrm{~min}$ before the procedure to prevent infection. A high-definition endoscope (GIF-Q260J; Olympus, Tokyo, Japan) with a transparent cap (D-201-11802; Olympus) was used. All procedures were performed with a carbon dioxide insufflator (UCR; Olympus), high-frequency generator (VIO 300D; ERBE, Tübingen, Germany), hybrid knife (JET2, APC2; ERBE), and endoscopic clips (HX-61090; Olympus).

Patients were placed in a supine position and administrated general anesthesia with endotracheal intubation. A submucosal injection with a mixture of methylene blue in saline solution $(0.2 \mathrm{ml}$ in $250 \mathrm{ml})$ was done approximately
$10 \mathrm{~cm}$ above the esophagogastric junction (EGJ) at the esophageal wall. A longitudinal mucosal entry with $6-8 \mathrm{~mm}$ in length was made. Then, a submucosal tunnel, occupying at least half of the esophageal lumen, was created 3-4 cm below the EGJ. Circular muscle myotomy was performed from $2-3 \mathrm{~cm}$ below the incision site down to $2-3 \mathrm{~cm}$ below the EGJ. Dual myotomy was performed at the 5-6 o'clock and 1-2 o'clock positions of the esophagus, respectively, which combined the two approaches for standard POEM. Finally, the mucosal entry was closed with endoscopic clips. (Fig. 1). The length of the myotomy was checked by withdrawing the endoscope from the submucosal tunnel into the stomach and observing the color change of the gastric mucosa with a retroflexed view. Complete myotomy could be confirmed by two indicators, including the loss resistance to passage of the endoscope at the EGJ, and observation of the EGJ opening with a retroflexed view at medium insufflation.

After the procedure, patients fasted for $24 \mathrm{~h}$. Intravenous antibiotics were continued for 1-3 days, and intravenous proton pump inhibitor (PPI) was maintained during the hospital stay. A liquid diet was allowed on postoperative days $2-3$ if there were no complications, and a soft diet was started within 2 weeks. An oral PPI was administrated for 2 weeks.

The initial follow-up visit was performed at approximately 3 months after dual-POEM. We assessed postoperative Eckardt score, upper gastrointestinal endoscopy, barium esophagography, high-resolution manometry, and reflux complications. Subsequent follow-up visit was then conducted annually to update the Eckardt score and estimate reflux complications. The final follow-up period ended in January 2021.

\section{Outcomes definition}

The primary outcome was clinical success, defined as an Eckardt score $\leq 3$ without an indication for retreatment, at follow-up assessment. The Eckardt score was used to rate the severity of achalasia-related symptoms, including weight loss, dysphagia, chest pain, and regurgitation [12]. Recurrence was defined as recurrent symptoms after initial success (Eckardt score $>3$ ). The indication for retreatment was that the patient had recurrence during the follow-up period.

Secondary outcomes included procedure-related adverse events, change in LES pressure, and reflux complications. Procedure-related adverse events were classified as minor or major according to their severity [13]. Reflux complications included symptomatic reflux and reflux esophagitis. Symptomatic reflux was defined as a score equal or greater than 8 in the gastroesophageal reflux disease questionnaire [14]. Reflux esophagitis was graded based on the Los Angeles classification [15]. Achalasia subtype was defined according to the Chicago classification [16]. Procedure time was 


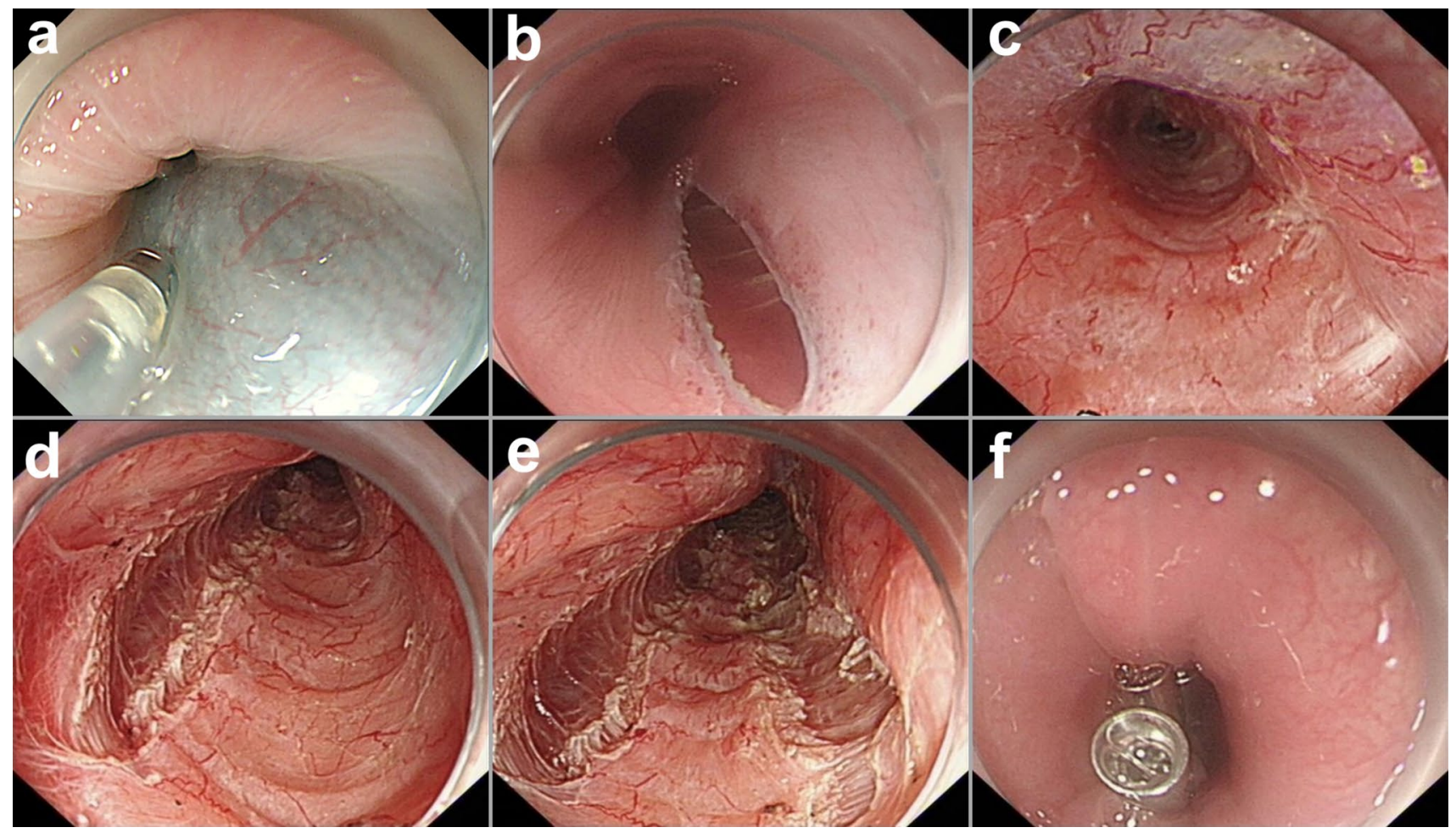

Fig. 1 Per-oral endoscopic dual myotomy (Dual-POEM). a A submucosal injection with a mixture of methylene blue in saline solution was done. b A longitudinal mucosal entry was made. c A wide submucosal tunnel, occupying at least half of the esophageal lumen,

recorded in minutes from submucosal injection to the end of dual-POEM. Hospital stay was the time that patients spent in the hospital after dual-POEM.

\section{Statistical analysis}

Continuous variables were expressed as mean (standard deviation, SD) or median with interquartile range (IQR) according to their distribution, whereas categorical variables were expressed as frequency or percentage. Median values between baseline and follow-up were compared using Mann-Whitney test. The statistical significance was set to $P<0.05$. All statistical analyses were conducted using the SPSS version 23.0 (IBM Corp., Armonk, New York, USA).

\section{Results}

\section{Patient characteristics}

During the study period, 17 patients ( 12 females, 5 males; mean age $43.2+11.5$ years; range $22-62$ ) with achalasia underwent dual-POEM and were regularly followed up. All of them were included in the final analysis (Table 1). was created. d The first myotomy was performed at the 5-6 o'clock position of the esophagus. e The second myotomy was performed at the 1-2 o'clock position of the esophagus. $\mathbf{f}$ The mucosal entry was closed with endoscopic clips

Table 1 Patient characteristics of 17 patients who underwent dualPOEM

\begin{tabular}{ll}
\hline Characteristics & Value \\
\hline Sex, female/male & $12 / 5$ \\
Age, years, mean (SD) & $43.2(11.5)$ \\
Duration of symptoms, years, median [IQR] & $4[3,12](1-20)$ \\
$\quad$ (range) & \\
Previous therapy, $n(\%)$ & $14(82.4)$ \\
None & $1(5.9)$ \\
Botulinum toxin injection & $2(11.8)$ \\
Balloon dilation & \\
Achalasia subtype, $n(\%)$ & $5(29.4)$ \\
Type I & $8(47.1)$ \\
Type II & $2(11.8)$ \\
Type III & $2(11.8)$ \\
No data & $4.2[3.7,5.2](3-6.2)$ \\
Maximum width, cm, median [IQR] (range) & \\
\hline
\end{tabular}

$S D$ standard deviation, $I Q R$ interquartile range

Prior to their dual-POEM, one patient had undergone botulinum toxin injection, and two had received balloon dilation. Twelve (70.6\%) patients had pretreatment Eckardt score $\geq 9$, 
and six (35.3\%) patients had $\geq 10$ years of symptoms. Fifteen patients underwent preprocedural manometry. The most common subtype of achalasia was type II $(8 / 17,47.1 \%)$. The median maximum width of the esophagus was $4.2 \mathrm{~cm}$ (IQR [3.7, 5.2]; range 3-6.2), and no patient had a sigmoid esophagus.

\section{Procedure-related parameters}

All procedures were successfully performed without technical difficulties. The median length of tunnel and myotomy were $10 \mathrm{~cm}$ (IQR [10, 12.5]; range 9-14) and $8 \mathrm{~cm}$ (IQR $[8,9.5]$; range 7-11), respectively. The median procedure time was 35 min (IQR [28, 45.5]; range 21-69). Procedurerelated adverse events were observed in $2(11.8 \%)$ patients and were classified as minor. One case of mucosal injury was successfully closed using endoscopic clips at the time of the procedure, without postoperative complications; another case of pneumonitis responded well to conservative treatment. Length of hospital stay was a median of 4 days (IQR [3, 4.5]; range 3-8).

\section{Follow-up outcomes}

At 3 months after dual-POEM, significant symptom relief and LES pressure reduction were noted in all 17 patients. The median Eckardt score decreased from 9 (IQR [8, 11.5]; range $7-12)$ to 1 (IQR $[0,1]$; range $0-3)(P<0.001)$ (Fig. 2a) and LES pressure decreased from $25.8 \mathrm{mmHg}$ (IQR [21.7, 33.5]; range 17.7-46.3) to 7.4 mmHg (IQR [6.3, 10.4]; range 2.2-12.6) $(P<0.001)$ (Fig. 2b). At 12-month follow-up, significant symptom relief was continued in all 17 patients, with a median Eckardt score of 1 (IQR $[1,1]$; range $0-3$ ). At further follow-up, one patient with achalasia type III developed recurrence. She reported recurrent symptoms with Eckardt score of 4 at 13 months. Subsequently, she underwent a salvage balloon dilation and no recurrence was observed on the 19-month follow-up assessment. During a median follow-up duration of 33 months (IQR [31, 35]; range 19-36), clinical success was achieved in $16(16 / 17$, 94.1\%) patients (Table 2; Fig. 3).

Three patients complained of symptomatic reflux, and two of them were found to have reflux esophagitis (one case of Los Angeles classification A and one case of Los Angeles classification B). Four asymptomatic patients had endoscopic evidence of esophagitis (Los Angeles classification A). The rates of symptomatic reflux and reflux esophagitis were $17.6 \%$ (3/17) and 35.3\% (6/17), respectively. The overall rate of reflux complications was $41.2 \%$ (7/17). In these patients, reflux symptoms typically presented at 3 months after dual-POEM, and reflux esophagitis was generally found on the 3-month follow-up endoscopy. These patients were treated with a standard dose of PPI and all achieved clinical response with symptom relief and endoscopic healing of esophagitis.

\section{Discussion}

In this study, we reported a novel technique, dual-POEM, for the treatment of achalasia in the selected group of patients with certain risk factors of POEM failure. No technical
Fig. 2 Symptom relief and lower esophageal sphincter (LES) pressure reduction before and after per-oral endoscopic dual myotomy. a Eckardt score. b LES pressure a

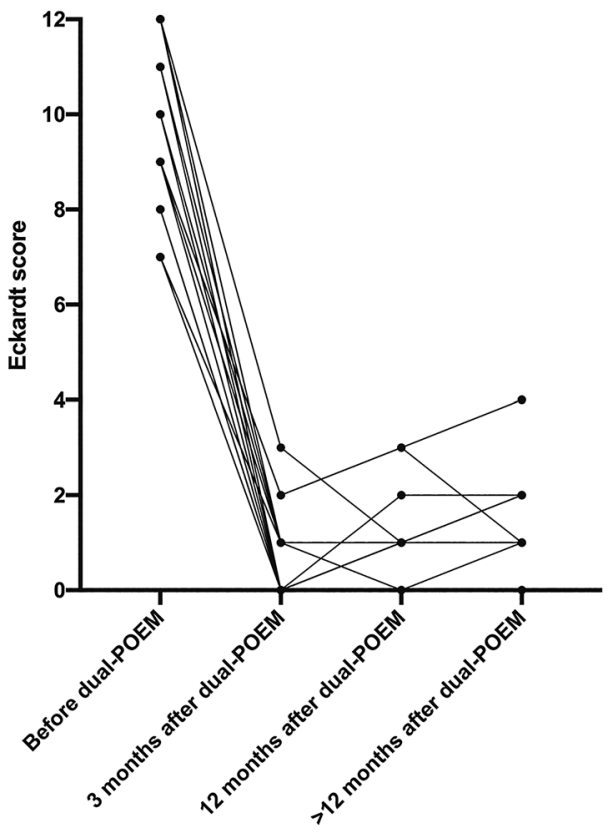

b

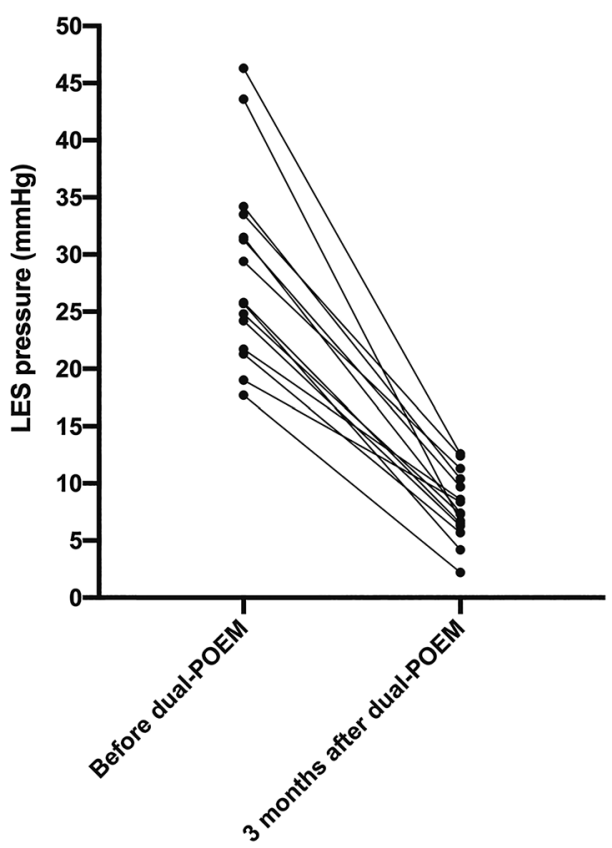


Table 2 Follow-up outcomes

\begin{tabular}{ll}
\hline Variable & Value \\
\hline Follow-up duration, months, median [IQR] (range) & 33 [31, 35] (19-36) \\
Reflux complications, $n(\%)$ & $7(41.2)$ \\
Symptomatic reflux only, $n(\%)$ & $1(5.9)$ \\
Endoscopic findings of esophagitis only, $n(\%)$ & $4(23.5)$ \\
Grade A & 4 \\
Symptomatic reflux and esophagitis on endoscopy, $n(\%)$ & $2(11.8)$ \\
Grade A & 1 \\
Grade B & 1 \\
Clinical success (Eckardt score $\leq 3), n(\%)$ & $16(94.1)$ \\
Recurrence (Eckardt score $>3), n(\%)$ & $1(5.9)$ \\
\hline
\end{tabular}

$I Q R$ interquartile range, POEM per-oral endoscopic myotomy, LES lower esophageal sphincter

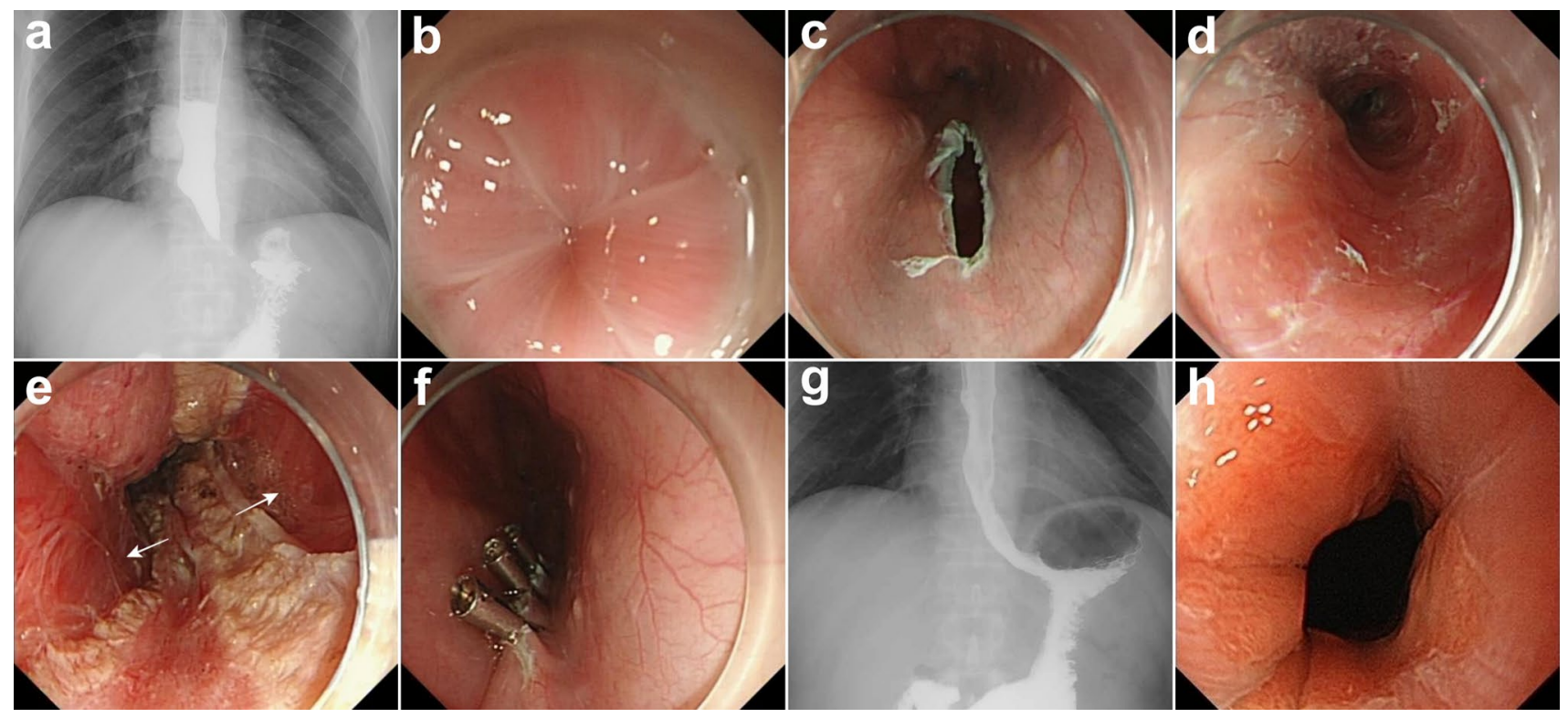

Fig. 3 A 36-year-old male with a 11-year history of achalasia. a No obvious passage of barium from the esophagus into the stomach. b A tightly closed cardia. c A longitudinal mucosal entry. d Creation of a wide submucosal tunnel. e Dual myotomy. f Closure of the mucosal

difficulties were observed during the dual-POEM procedures; thus, dual-POEM appears to be technically feasible. All patients but one (94.1\%) maintained a significant clinical response, achieving posttreatment Eckardt score $\leq 3$ at the median follow-up duration of 33 months. No major dualPOEM-related adverse events and no serious reflux complications were observed. Dual-POEM proved to be safe and effective.

Although POEM is considered to be a highly effective treatment for achalasia, POEM failure does occur. Several mid-term studies have shown that the rate of POEM failure ranges from 5.8 to $21.5 \%$ [5-8], and increases over time. The efficacy of different retreatments has been extensively entry. g Postoperative barium esophagography indicated a rapid passage of barium. h Follow-up upper gastrointestinal endoscopy revealed a relaxed cardia

studied. Balloon dilation showed a lower clinical successful rate $(0-22 \%)[5,7]$, whereas retreatment with POEM or LHM yielded better outcomes $(40-100 \%)[5,7,9,10]$. This suggests that repeat myotomy is a more effective remedy after initial POEM failure. Based on this finding, dualPOEM might be a promising method with the potential advantage of avoiding repeat operations. The results of the present series preliminarily confirmed this hypothesis. In our study, in patients having more than one risk factors for achalasia recurrence after initial POEM, clinical success rate was $94.1 \%$ during a median follow-up of 33 months. Even though this success rate is close to the ones for standard POEM, the available success rates on standard POEM 
were obtained from patients with lower pretreatment Eckardt score in general. Our study has looked at a special population with more than one risk factors for treatment failure including higher pretreatment Eckardt score, long disease duration and prior treatments for achalasia, which are usually associated with more severe or refractory disease. A study by Werner et al. [5] has shown that the clinical success rate was around $80 \%$ in patients undergoing POEM at 2-year follow-up. Our dual-POEM success rate is better than one reported by Werner; this suggests that dual-POEM may indeed have the advantage in mitigating the risk of recurrence, avoiding a repeat procedure. As for the patient (type III) who developed recurrence, a different pathophysiology from a different manometric subtype is one of the likely explanations [17]. However, given our limited number of patients and relatively short follow-up time, further studies with longer follow-up duration are needed to clarify this phenomenon in type III achalasia.

Regarding the safety of dual-POEM, although the rate of dual-POEM-related adverse events $(11.8 \%)$ was relatively high when compared with that of standard POEM [18], these adverse events were minor and could be managed conservatively. Mucosal injury was managed successfully with endoscopic clipping. Mild pneumonitis was controlled with administration of antibiotics. Pneumonitis may have been caused by inadequate preoperative esophageal cleaning; thus, preoperative preparation in addition to the use of prophylactic antibiotics and minimizing aspiration should be well executed to reduce the possibility for such infections [19]. In our study, both patients with dual-POEMrelated adverse events recovered quickly without apparent deleterious clinical outcomes. Of course, our limited sample size may also have artificially inflated the rate of adverse events. As with standard POEM, gastroesophageal reflux was the major complication of dual-POEM. Previous studies have reported that the rate of symptomatic reflux and reflux esophagitis after standard POEM ranges from 9 to $39.9 \%$ [5, 8, 20-22] and 14.8-66\% [3, 6, 8, 20], respectively. Comparable results were noted in dual-POEM. In our series, no patients developed reflux esophagitis of more than Los Angeles classification B. All patients who had reflux were successfully treated with PPIs, and none required rescue fundoplication. Dual-POEM does not seem to increase posttreatment reflux disease.

An interesting issue for discussion is on which side dualPOEM should be done. During the standard POEM procedure, two approaches are proposed: anterior and posterior myotomy. Anterior myotomy at the 1-2 o'clock position of the esophagus ensures the minimum curvature of the submucosal tunnel to the stomach and avoids damage to the angle of His (positioned at the 8 o'clock), which is an important natural anti-reflux mechanism [23]. Posterior myotomy at the 5-6 o' clock position enables better alignment of the knife during tunnel creation and myotomy [23]. Investigations indicated that the efficacy and safety of each approach are comparable [23, 24]; thus, endoscopists could choose either approach according to their preferences. Based on these, our dual myotomy was performed at the 1-2 o'clock and 5-6 o'clock positions, respectively. The endoscopist in this study has an extensive experience in POEM; thus, there were no technical difficulties during tunnel creation and dual myotomy. However, the technical feasibility of dual-POEM still needs to be studied among the endoscopists with early POEM experience.

There were some limitations in this study, including its retrospective design, small sample size, a single operator and noncomparative design. Additionally, due to the locally limited accessibility, objective testing such as 24-h esophageal $\mathrm{pH}$ measurements was lacking. Although the major limitation seems to be its retrospective design, the data collected from a prospectively collected database supports the validity of the present results.

In conclusion, we report the first case series with favorable results from dual-POEM for the treatment of achalasia in selected patients. Dual-POEM might be a promising option for patients presenting with risk factors of POEM failure. Multicenter, prospective comparative studies with a larger sample size are needed to clarify its indications and to further evaluate its safety and efficacy.

Acknowledgements We acknowledge the support from 1.3.5 project for disciplines of excellence-Clinical Research Incubation Project, West China Hospital, Sichuan University (Grant No: 20HXFH016).

\section{Declarations}

Ethical Statement This study protocol was approved by the Ethics Committee on Biomedical Research, West China Hospital of Sichuan University.

Conflicts of interest Part of the abstract of this manuscript has been accepted for an E-poster presentation (oral) at Asian Pacific Digestive Week (APDW), held in Kolkata, India, 12th-15th December 2019. Authors disclose no conflicts relevant to this article.

Open Access This article is licensed under a Creative Commons Attribution 4.0 International License, which permits use, sharing, adaptation, distribution and reproduction in any medium or format, as long as you give appropriate credit to the original author(s) and the source, provide a link to the Creative Commons licence, and indicate if changes were made. The images or other third party material in this article are included in the article's Creative Commons licence, unless indicated otherwise in a credit line to the material. If material is not included in the article's Creative Commons licence and your intended use is not permitted by statutory regulation or exceeds the permitted use, you will need to obtain permission directly from the copyright holder. To view a copy of this licence, visit http://creativecommons.org/licenses/by/4.0/. 


\section{References}

1. Vaezi MF, Pandolfino JE, Vela MF. ACG clinical guideline: diagnosis and management of achalasia. Am J Gastroenterol. 2013;108:1238-49.

2. Inoue $\mathrm{H}$, Minami $\mathrm{H}$, Kobayashi $\mathrm{Y}$, et al. Peroral endoscopic myotomy (POEM) for esophageal achalasia. Endoscopy. 2010;42:265-71.

3. Inoue $\mathrm{H}$, Sato $\mathrm{H}$, Ikeda $\mathrm{H}$, et al. Per-oral endoscopic myotomy: a series of 500 patients. J Am Coll Surg. 2015;221:256-64.

4. Park CH, Jung DH, Kim DH, et al. Comparative efficacy of peroral endoscopic myotomy and Heller myotomy in patients with achalasia: a meta-analysis. Gastrointest Endosc. 2019;90:546-58.

5. Werner YB, Costamagna G, Swanstrom LL, et al. Clinical response to peroral endoscopic myotomy in patients with idiopathic achalasia at a minimum follow-up of 2 years. Gut. 2016;65:899-906.

6. Ren Y, Tang X, Chen Y, et al. Pre-treatment Eckardt score is a simple factor for predicting one-year peroral endoscopic myotomy failure in patients with achalasia. Surg Endosc. 2017;31:3234-41.

7. van Hoeij FB, Ponds FA, Werner Y, et al. Management of recurrent symptoms after per-oral endoscopic myotomy in achalasia. Gastrointest Endosc. 2018;87:95-101.

8. Li QL, Wu QN, Zhang XC, et al. Outcomes of per-oral endoscopic myotomy for treatment of esophageal achalasia with a median follow-up of 49 months. Gastrointest Endosc. 2018;87(6):1405-12.

9. Li QL, Yao LQ, Xu XY, et al. Repeat peroral endoscopic myotomy: a salvage option for persistent/recurrent symptoms. Endoscopy. 2016;48:134-40.

10. Tyberg A, Seewald S, Sharaiha RZ, et al. A multicenter international registry of redo per-oral endoscopic myotomy (POEM) after failed POEM. Gastrointest Endosc. 2017;85:1208-11.

11. Yuan XL, Liu W, Ye LS, et al. Peroral endoscopic dual myotomy (dual POEM) for achalasia with severe esophageal dilatation. Endoscopy. 2018;50:E179-80.

12. Eckardt AJ, Eckardt VF. Treatment and surveillance strategies in achalasia: an update. Nat Rev Gastroenterol Hepatol. 2011;8:311-9.

13. Nabi Z, Reddy DN, Ramchandani M. Adverse events during and after per-oral endoscopic myotomy: prevention, diagnosis, and management. Gastrointest Endosc. 2018;87:4-17.

14. Jones R, Junghard O, Dent J, et al. Development of the GerdQ, a tool for the diagnosis and management of gastro-oesophageal reflux disease in primary care. Aliment Pharmacol Ther. 2009;30:1030-8.

15. Armstrong D, Bennett JR, Blum AL, et al. The endoscopic assessment of esophagitis: a progress report on observer agreement. Gastroenterology. 1996;111:85-92.

16. Kahrilas PJ, Bredenoord AJ, Fox M, et al. The Chicago Classification of esophageal motility disorders, v3.0. Neurogastroenterol Motil. 2015;27:160-74.

17. Hernandez Mondragon OV, Gonzalez Martinez MA, Blancas Valencia JM, et al. Long-term quality of life after peroral endoscopic myotomy remains compromised in patients with achalasia type III. Endoscopy. 2017;49:1209-18.

18. Haito-Chavez Y, Inoue H, Beard KW, et al. Comprehensive analysis of adverse events associated with per oral endoscopic myotomy in 1826 patients: an international multicenter study. Am J Gastroenterol. 2017;112:1267-76.

19. Ren Z, Zhong Y, Zhou P, et al. Perioperative management and treatment for complications during and after peroral endoscopic myotomy (POEM) for esophageal achalasia (EA) (data from 119 cases). Surg Endosc. 2012;26:3267-72.

20. Shiwaku H, Inoue H, Yamashita K, et al. Peroral endoscopic myotomy for esophageal achalasia: outcomes of the first over 100 patients with short-term follow-up. Surg Endosc. 2016;30:4817-26.

21. Kumbhari V, Familiari P, Bjerregaard NC, et al. Gastroesophageal reflux after peroral endoscopic myotomy: a multicenter casecontrol study. Endoscopy. 2017;49:634-42.

22. Jones EL, Meara MP, Schwartz JS, et al. Gastroesophageal reflux symptoms do not correlate with objective $\mathrm{pH}$ testing after peroral endoscopic myotomy. Surg Endosc. 2016;30:947-52.

23. Tan Y, Lv L, Wang X, et al. Efficacy of anterior versus posterior per-oral endoscopic myotomy for treating achalasia: a randomized, prospective study. Gastrointest Endosc. 2018;88:46-54.

24. Rodriguez de Santiago E, Mohammed N, Manolakis A, et al. Anterior versus posterior myotomy during poem for the treatment of achalasia: systematic review and meta-analysis of randomized clinical trials. J Gastrointest Liver Dis. 2019;28:107-15.

Publisher's Note Springer Nature remains neutral with regard to jurisdictional claims in published maps and institutional affiliations. 